\title{
A study on clinicopathological features and prognostic factors of patients with upper gastric cancer and middle and lower gastric cancer.
}

\author{
Gang Wang", Jingrong Zhou\#, Feng Lu, Lei Qiu, Yongchang Miao* \\ The Second People's Hospital of Lianyungang, Haizhou Sea East Road No. 41, Jiangsu Province, PR China \\ \#These authors contributed equally to this work
}

\begin{abstract}
Objective: To compare the difference on clinicopathological features and prognostic factors in patients with upper gastric cancer and middle and lower gastric cancer.

Methods: 168 cases of gastric cancer patients treated in our hospital from January 2010 to March 2013 were enrolled as the research objects. All of these patients have complete data and were confirmed by pathology and classified into stages I-III period, with 90 cases of upper gastric cancer and 78 cases of middle and lower gastric cancer respectively. Of the 168 cases, 150 cases $(89.3 \%)$ underwent Ro treatment (no residue under microscope after resection), 18 cases $(10.7 \%)$ underwent $R 1$ treatment (microscopic residual) and 152 cases $(\mathbf{9 0 . 5 \%})$ underwent D2 (radical type II) perigastric lymph node dissection treatment.

Results: There were no significant differences on TNM stage, operation modes, the number of dissected lymph nodes and postoperative complications between the upper gastric cancer group and middle and lower gastric cancer group $(\mathbf{P}>\mathbf{0 . 0 5})$. There were significant differences on preoperative complications and postoperative adjuvant chemotherapy between the upper gastric cancer group and middle and lower gastric cancer group $(\mathbf{P}<\mathbf{0 . 0 5}) .3$ y Overall Survival $(\mathrm{OS})$ and Progression-Free Survival $(\mathrm{PFS})$ in the upper gastric cancer group were $35.6 \%$ (32/90 cases) and $47.8 \%(43 / 90)(P=0.026)$, and $43.6 \%(34 / 78$ cases) and 51.3\% (40/78) respectively in middle and lower gastric cancer group ( $P=0.035)$. Logistic regression analysis showed that risk factors affecting the prognosis of upper gastric cancer were preoperative complications and postoperative adjuvant chemotherapy, while the risk factors affecting the prognosis of middle and lower gastric cancer were TNM staging, preoperative complications and postoperative adjuvant chemotherapy. By the last follow-up on March 31, 2017, 51 of $90(56.7 \%)$ patients with upper gastric cancer and 34 of $78(43.6 \%)$ patients with middle and lower gastric cancer died respectively $(\mathbf{P}<\mathbf{0 . 0 5})$.

Conclusion: There were significant differences on the clinical pathological features and prognosis between patients with upper gastric cancer and middle and lower gastric cancer patients, it is of great guiding significance to know the pathological features of cancer in different parts, it can provide individualized treatment options for patients and improve the prognosis of patients.
\end{abstract}

Keywords: Gastric cancer, Upper gastric cancer, Middle and lower gastric cancer, Pathological features, Prognostic factors.

Accepted on October 30, 2017

\section{Introduction}

Gastric cancer is one of the most common malignant tumors, and its fatality rate ranks the third [1] of all malignant tumors. China is the main country of gastric cancer in Asia, accounting for $42.6 \%$ of the patients the entire world and the incidence of elderly gastric cancer in China has increased significantly in recent years. Despite the significant progress of gastric cancer treatment (such as surgery, radiotherapy, chemotherapy and targeted therapy) in recent years, the 5 y survival rate is only $20 \%-30 \%$, which was closely related to the high malignancy and biological complexity of gastric cancer [2]. Upper gastric cancer mainly includes lesions at the junction and the fundus of the stomach, and upper gastric cancer is usually diagnosed in the advanced stage. Due to complexed lymphatic drainage in this area and the specific operation methods which involves thoracotomy, gastrointestinal anastomosis of esophagus and diaphragm, abdominal blood vessels and lymph node removal, the efficacy of treatment is often poor [3]. Previous studies have shown that [4], upper gastric cancer has unique epidemiological and biological characteristics, and surgical treatment is different from the middle and lower gastric cancer. It was also reported that [5], compared with middle and lower gastric cancer, there were more male patients than female 
patients with upper gastric cancer. Upper gastric cancer is often a late disease with low survival rate and is pathologically characterized by diffuse growth and low grade. Relevant data show that $[6,7]$ the prognosis of gastric cancer is not only closely related to the treatment methods, biological behaviors and clinical pathological features, but also affected by gross type, invasion depth, lymph node metastasis and infiltration growth types and types of gastric cancer serosal surface. At present, there are few reports about the differences of prognostic factors between upper gastric cancer and middle and lower gastric cancer. Our previous studies have shown that the prognosis of upper gastric cancer may be poor. Therefore, this study further compared the clinicopathological features, prognosis and prognostic factors between patients with upper gastric cancer and middle and lower gastric cancer.

\section{Data and Methods}

\section{General data}

168 cases of gastric cancer patients treated in our hospital from January 2010 to March 2013 were enrolled as the research objects. All of these patients have complete data and were confirmed by pathology and classified into stages I-III period. General data were as follows: 116 males and 52 females; aged 28 to $76 \mathrm{y}$ old with a mean age $(64.7 \pm 1.5 \mathrm{y}) ; 132$ cases of adenocarcinoma, 34 cases of signet ring cell carcinoma and 2 cases of adenosquamous carcinoma; 90 cases of upper gastric cancer and 78 cases of middle and lower gastric cancer. All patients routinely underwent gastroscopy, upper abdominal CT, chest X-ray, ultrasound examination of neck and other staging examinations, and were confirmed no distant metastasis. Electrocardiogram, blood routine, liver and kidney function examination were routinely performed before operation and chemotherapy. There were clear indications for surgery treatment and postoperative adjuvant chemotherapy, and all the patients were informed before the treatment and signed consents.

\section{Treatment modes}

Of 168 patients with gastric cancer, 150 (89.3\%) underwent $\mathrm{R}_{0}$ treatment, $18(10.7 \%)$ underwent $\mathrm{R}_{1}$ treatment, and 152 (90.5\%) underwent $\mathrm{D}_{2}$ peri-gastric lymph node dissection treatment. The number of dissected perigastric lymph nodes was 3 to 50, and the median was 20. Among these patients, $74.4 \%(125 / 168)$ patients underwent $>15$ lymph nodes dissection. The number of positive lymph nodes was $0-38$, and the median was 6 . 64 cases $(38.1 \%)$ underwent adjuvant chemotherapy for 1-8 cycles, with a median of 4 cycles.

\section{Follow up}

All patients were reexamined every 3 months within 2 y after the first course of treatment, and every 6 months within 3 to 5 y. Routine examination included physical examination, routine blood test, liver and kidney function and imaging examinations. Patients were followed by telephone. The primary endpoints were Overall Survival (OS) and Progression
Free Survival (PFS). The causes of death were defined as cancer related deaths, cancer treatment related deaths and comorbidities related deaths.

\section{Statistical analysis}

SPSS 21 statistical software was adopted for analysis. Comparisons on the rate differences between the two groups were conducted using the Chi-square test. Survival rates were analysed using Kaplan-Meier. Survival difference between the groups was compared using log-rank test. Multivariate analysis was analysed using Logistic regression analysis. $\mathrm{P}<0.05$ means a significant difference.

\section{Results}

\section{Comparisons on basic clinical characteristics between upper gastric cancer and middle and lower gastric cancer}

There were no significant differences on basic clinical characteristics of sex, age, size of cancer and so on between the upper gastric cancer group and middle and lower gastric cancer $\operatorname{group}(\mathrm{P}>0.05$, Table 1$)$.

Table 1. Comparisons on basic clinical characteristics between upper gastric cancer and middle and lower gastric cancer.

\begin{tabular}{|c|c|c|c|}
\hline $\begin{array}{l}\text { Basic clinical } \\
\text { characteristics }\end{array}$ & $\begin{array}{l}\text { Upper gastric } \\
\text { cancer }(n=90)\end{array}$ & $\begin{array}{l}\text { Middle and lower } \\
\text { gastric cancer }(n=78)\end{array}$ & $\mathbf{P}$ \\
\hline Sex & & & 0.095 \\
\hline Male & 62 & 44 & \\
\hline Female & 28 & 34 & \\
\hline Age (y) & $63.9 \pm 1.4$ & $65.1 \pm 1.7$ & 0.264 \\
\hline Size of cancer & & & 0.899 \\
\hline$<5 \mathrm{~cm}$ & 72 & 63 & \\
\hline$\geq 5 \mathrm{~cm}$ & 18 & 15 & \\
\hline Growth pattern & & & 0.131 \\
\hline Clumps growth & 9 & 16 & \\
\hline Nests growth & 29 & 19 & \\
\hline Diffuse growth & 52 & 43 & \\
\hline Depth of invasion & & & 0.127 \\
\hline T1 & 9 & 12 & \\
\hline $\mathrm{T} 2$ & 21 & 26 & \\
\hline T3 & 60 & 40 & \\
\hline $\begin{array}{l}\text { Pathological } \\
\text { classification }\end{array}$ & & & 0.306 \\
\hline Glandular cancer & 70 & 62 & \\
\hline $\begin{array}{l}\text { Signet ring cell } \\
\text { carcinoma }\end{array}$ & 19 & 15 & \\
\hline
\end{tabular}


A study on clinicopathological features and prognostic factors of patients with upper gastric cancer and middle and lower gastric cancer

\section{Gland scale cancer $\quad 0$ \\ Comparisons on clinicopathological features and treatment modes between upper gastric cancer and middle and lower gastric cancer}

There were no significant differences on TNM stage, operation modes, the number of dissected lymph nodes and postoperative complications between the upper gastric cancer group and middle and lower gastric cancer group $(\mathrm{P}>0.05)$. There were significant differences on preoperative complications and postoperative adjuvant chemotherapy between the upper gastric cancer group and middle and lower gastric cancer group $(\mathrm{P}<0.05$, Table 2).

Table 2. Comparisons on clinicopathological features between upper gastric cancer and middle and lower gastric cancer.

\begin{tabular}{|c|c|c|c|c|}
\hline $\begin{array}{l}\text { Clinicopathological } \\
\text { features }\end{array}$ & $\begin{array}{l}\text { Upper gastric } \\
\text { cancer }(n=90)\end{array}$ & $\begin{array}{l}\text { Lower gastric } \\
\text { cancer }(n=78)\end{array}$ & $x^{2}$ & $\mathbf{P}$ \\
\hline TNM staging & & & 0.672 & 0.731 \\
\hline I-II & 30 & 38 & & \\
\hline III & 60 & 40 & & \\
\hline \multicolumn{5}{|l|}{$\begin{array}{l}\text { Preoperative } \\
\text { complications }\end{array}$} \\
\hline Yes & 46 & 30 & 12.518 & 0.000 \\
\hline No & 44 & 48 & & \\
\hline Operation modes 1 & & & & 0.606 \\
\hline $\mathrm{R}_{0}$ & 80 & 70 & & \\
\hline $\mathrm{R}_{1}$ & 10 & 8 & & \\
\hline Operation modes 2 & & & 1.084 & 0.472 \\
\hline $\mathrm{D}_{0} / \mathrm{D}_{1}$ & 8 & 4 & & \\
\hline $\mathrm{D}_{2}$ & 82 & 74 & & \\
\hline
\end{tabular}

\begin{tabular}{|c|c|c|c|c|}
\hline $\begin{array}{l}\text { Dissected } \\
\text { nodes }\end{array}$ & lymph & & 0.103 & 0.146 \\
\hline$<15$ & 20 & 23 & & \\
\hline$\geq 15$ & 70 & 55 & & \\
\hline $\begin{array}{l}\text { Postoperative } \\
\text { complications }\end{array}$ & & & 0.455 & 0.593 \\
\hline Yes & 9 & 6 & & \\
\hline No & 81 & 72 & & \\
\hline $\begin{array}{l}\text { Postoperative } \\
\text { adjuvant } \\
\text { chemotherapy }\end{array}$ & & & 10.169 & 0.007 \\
\hline Yes & 50 & 14 & & \\
\hline No & 40 & 64 & & \\
\hline
\end{tabular}

\section{Comparisons on prognosis between patients with upper gastric cancer and middle and lower gastric cancer}

By the last follow-up, the total follow-up was 1 to 40 months with a median follow-up of 24.5 months. 3 y OS and PFS in patients with upper gastric cancer were $35.6 \%$ (32/90 cases) and $47.8 \%(43 / 90)$, respectively $(\mathrm{P}=0.026)$, and $43.6 \%(34 / 78$ cases) and $51.3 \%$ (40/78 cases) in middle and lower gastric cancer patients $(\mathrm{P}=0.035)$.

\section{Multivariate analysis of prognostic factors between patients with upper gastric cancer and middle and lower gastric cancer}

Logistic regression analysis showed that risk factors affecting the prognosis of upper gastric cancer were preoperative complications and postoperative adjuvant chemotherapy, while the risk factors affecting the prognosis of middle and lower gastric cancer were TNM staging, preoperative complications and postoperative adjuvant chemotherapy (Table 3 ).

Table 3. Multivariate analysis of prognostic factors between patients with upper gastric cancer and middle and lower gastric cancer.

\begin{tabular}{|c|c|c|c|c|c|}
\hline Cancer site & B & SE & Wald $\mathrm{x}^{2}$ & OR (95\% CI) & $\mathbf{P}$ \\
\hline \multicolumn{6}{|l|}{ Upper gastric cancer } \\
\hline TNM staging & 0.234 & 0.502 & 0.308 & 1.344 & 0.604 \\
\hline (I+II vs. III) & & & & $(0.501-3.154)$ & \\
\hline Preoperative complications & 2.057 & 0.535 & 13.608 & 7.509 & $<0.001$ \\
\hline (No vs. Yes) & & & & $(2.682-22.146)$ & \\
\hline Postoperative adjuvant chemotherapy & 0.724 & 0.261 & 6.703 & 1.744 & 0.018 \\
\hline (No vs. Yes) & & & & $(1.563-2.951)$ & \\
\hline \multicolumn{6}{|l|}{ Lower gastric cancer } \\
\hline TNM staging & 0.416 & 0.065 & 29.182 & 1.686 & 0 \\
\hline (I+II vs. III) & & & & $(0.957-4.132)$ & \\
\hline
\end{tabular}




\begin{tabular}{llllll}
\hline Preoperative complications & -1.232 & 0.504 & 6.612 & 0.255 & 0.017 \\
\hline (No vs. Yes) & & & & $(0.091-0.741)$ & 0.006 \\
\hline Postoperative adjuvant chemotherapy & 1.406 & 0.603 & 7.115 & 4.026 & $(1.539-13.425)$ \\
\hline (No vs. Yes) & & & & \\
\hline
\end{tabular}

\section{Comparisons on death causes in patients with upper gastric cancer and middle and lower gastric cancer}

By the last follow-up on March 31, 2017, 51 of 90 (56.7\%) patients with upper gastric cancer and 34 of 78 (43.6\%) patients with middle and lower gastric cancer died respectively
$(\mathrm{P}<0.05)$. Among these deaths, cancer related death was 44 and 32 cases respectively in the two groups, while cancer treatment or complication related death was 7 and 2 cases respectively (Table 4).

Table 4. Comparisons on death causes in patients with upper gastric cancer and middle and lower gastric cancer.

\begin{tabular}{llll}
\hline Death causes & Death in upper gastric cancer $(\mathbf{n = 5 1 )}$ & Death in middle and lower gastric cancer (n=34) & $\mathbf{P}$ \\
\hline Cancer related deaths & 44 & 32 & 0.127 \\
\hline $\begin{array}{l}\text { Treatment or comorbidities } \\
\text { related deaths }\end{array}$ & 7 & 2 & 0.024 \\
\hline Pulmonary infection & 2 & 0 \\
\hline Cardiovascular accident & 2 & 0 \\
\hline Anastomotic bleeding & 1 & 1 \\
\hline Intestinal obstruction & 1 & 0 \\
\hline Anastomotic obstruction & 1 & 1 \\
\hline
\end{tabular}

\section{Summary}

There were no significant differences on TNM stage, operation modes, the number of dissected lymph nodes and postoperative complications between the upper gastric cancer group and middle and lower gastric cancer group $(\mathrm{P}>0.05)$. There were significant differences on preoperative complications and postoperative adjuvant chemotherapy between the upper gastric cancer group and middle and lower gastric cancer group $(\mathrm{P}<0.05) .3$ y OS and PFS in the upper gastric cancer group were $35.6 \%$ (32/90 cases) and $47.8 \%(43 / 90)(\mathrm{P}=0.026)$, and $43.6 \%$ (34/78 cases) and $51.3 \%(40 / 78)$ respectively in middle and lower gastric cancer group $(\mathrm{P}=0.035)$. Logistic regression analysis showed that risk factors affecting the prognosis of upper gastric cancer were preoperative complications and postoperative adjuvant chemotherapy, while the risk factors affecting the prognosis of middle and lower gastric cancer were TNM staging, preoperative complications and postoperative adjuvant chemotherapy. By the last follow-up on March 31, 2017,51 of $90(56.7 \%)$ patients with upper gastric cancer and 34 of $78(43.6 \%)$ patients with middle and lower gastric cancer died respectively $(\mathrm{P}<0.05)$.

\section{Discussion}

From the etiological perspective, gastric cancer is the result of many factors, the pathogenesis involves changes in genetics of many genes and pathways and epigenetic changes, and it shows different trends in various clinical stages. In China, the incidence and mortality of gastric cancer are at the forefront of malignant tumors. Although some progress has been made in the basic and clinical research of gastric cancer in recent decades, the diagnosis and treatment of gastric cancer is still not optimistic, the $5 \mathrm{y}$ overall survival rate is still low. Most of the gastric cancer is already in the middle and late stage when diagnosed, about $60 \%$ of patients still have the recurrence and metastasis even comprehensive treatment based on surgery treatment has been performed $[8,9]$.

Previous studies have confirmed that [10], the site of gastric cancer is an independent prognostic factor. In this study, we analysed clinical data of patients with upper gastric cancer and middle and lower gastric cancer to investigate the pathological features of cancer at different parts and to investigate the clinical significance of the differences, which has important significance in the individual treatment for gastric cancer. Some scholars [11] have found that the proportion of male gastric cancer patients is significantly higher than that of female, and the age composition of gastric cancer is the least in young people and the most in the elderly, which is basically consistent with the results of this study.

The biology of tumor is an important basis for the occurrence, development and clinical pathological characteristics, reflecting the nature or malignant degree of a tumor [12]. The biological behaviors of gastric cancer in different stages are significantly different. The results of this study showed that there were no significant differences on TNM stage, operation 
modes, the number of dissected lymph nodes and postoperative complications between the upper gastric cancer group and middle and lower gastric cancer group $(\mathrm{P}>0.05)$. There were significant differences on preoperative complications and postoperative adjuvant chemotherapy between the upper gastric cancer group and middle and lower gastric cancer group $(\mathrm{P}<0.05)$. It is suggested that there are significant differences in preoperative complications and postoperative adjuvant chemotherapy and other clinical pathological features between upper gastric cancer and middle and lower gastric cancer.

The incidence of upper gastric cancer is increasing in recent years. Studies have shown that [13-15] upper gastric cancer is difficult to be detected in early stage, and is characterized by low degree of differentiation, high degree of malignancy, wide invasion and other pathological features. The prognosis is significantly worse than the middle and lower gastric cancer. The results of this study showed that the $3 \mathrm{y}$ OS and PFS of patients with upper gastric cancer were significantly lower than those of patients with middle and lower gastric cancer $(\mathrm{P}<0.05)$. The main reason of the worse prognosis of patients with upper gastric cancer is that the early symptoms are not obvious, the related lesions mostly grow infiltratively or even spread directly, causing more extensive and subtle lymph node metastasis. Special anatomic sites and more residual cancer can also lead to the result. Logistic regression analysis showed that risk factors affecting the prognosis of upper gastric cancer were preoperative complications and postoperative adjuvant chemotherapy, while the risk factors affecting the prognosis of middle and lower gastric cancer were TNM staging, preoperative complications and postoperative adjuvant chemotherapy. It is suggested that there may be some differences in the clinical prognostic factors between upper gastric cancer and middle and middle and lower gastric cancer. From the statistical results, we speculated that the upper gastric cancer is characterized by low differentiation, late clinical stage, deep invasion and extensive lymph node metastasis. These clinical features determine their prognosis. To improve the early diagnosis rate of gastric cancer, and to follow the standard radical operation standard actively, it is helpful to improve the curative effect of gastric cancer by comprehensive treatment based on operation.

In summary, there were significant differences on the clinical pathological features and prognosis between patients with upper gastric cancer and patients with middle and lower gastric cancer, it is of great guiding significance to know the pathological features of cancer in different parts, it can provide individualized treatment options for patients and improve the prognosis of patients. As our study is retrospective and the sample size was relatively small, there may be some bias in the study, and a prospective study with larger sample size is needed to confirm the findings.

\section{References}

1. Sano T, Coit DG, Kim HH. Proposal of a new stage grouping of gastric cancer for TNM classification:
International Gastric Cancer Association staging project. Gastric Cancer 2017; 20: 1-9.

2. Shoda K, Ichikawa D, Fujita Y. Monitoring the HER2, copy number status in circulating tumor DNA by droplet digital PCR in patients with gastric cancer. Gastric Cancer 2017; 20: $1-10$.

3. Chen K, Pan Y, Zhang B. Robotic versus laparoscopic Gastrectomy for gastric cancer: a systematic review and updated meta-analysis. BMC Surg 2017; 17: 93.

4. Cheng Z, Li M, Liu J. Integrated analysis identified an intestinal-like and a diffuse-like gene sets that predict gastric cancer outcome. Tumor Biol 2016; 1-19.

5. Kim SH, Lee SH, Choi YL. Extensive alteration in the expression profiles of TGFB pathway signaling components and TP53 is observed along the gastric dysplasia-carcinoma sequence. Histol Histopathol 2008; 23: 1439-1452.

6. Lu J, Huang CM, Zheng $\mathrm{CH}$. Analysis on the clinical and pathological features and prognosis of familial gastric cancer in South China population: a single-center study of 724 patients. J Oncol 2012; 641218.

7. Hsu JT, Chen TD, Chuang HC. Vascular endothelial growth factor expression is an independent poor prognostic factor for HER-2 positive gastric cancer. J Surg Res 2017; 208: 40-50.

8. Kaku M, Mathew A, Rajan B. Impact of socio-economic factors in delayed reporting and late-stage presentation among patients with cervix cancer in a major cancer hospital in South India. Asian Pac J Cancer Prev 2008; 9: 589-594.

9. Ji YC, Ha TK, Kwon SJ. Clinicopathologic characteristics of gastric cancer patients according to the timing of the recurrence after curative surgery. J Gastric Cancer 2011; 11: 46-54.

10. Nitti D, Marchet A, Olivieri M. Ratio between metastatic and examined lymph nodes is an independent prognostic factor after D2 resection for gastric cancer: analysis of a large European monoinstitutional experience. Ann Surg Oncol 2003; 10: 1077-1085.

11. Mrena J, Wiksten J P, Thiel A. Cyclooxygenase-2 is an independent prognostic factor in gastric cancer and its expression is regulated by the messenger RNA stability factor HuR. Clinic Off J Am Assoc Cancer Res 2005; 11: 7362-7368.

12. Lu X, Cheng C, Zhu S. SATB1 is an independent prognostic marker for gastric cancer in a Chinese population. Oncol Rep 2010; 24: 981-987.

13. Salehi Z, Mollasalehi H, Jelodar MH. The relationship between Helicobacter pylori infection and gastric adenocarcinoma in northern Iran. Oncol Res Feat Preclinic Clinic Cancer Ther 2010; 18: 323-328.

14. Sakakibara T, Hibi K, Koike M. Plasminogen activator inhibitor-1 as a potential marker for the malignancy of gastric cancer. Cancer Sci 2006; 97: 395-399.

15. Lee DJ, Sohn TS, Lim DH. Phase I study of neoadjuvant chemoradiotherapy with S-1 and oxaliplatin in patients with 
locally advanced gastric cancer. Cancer Chemother Pharmacol 2012; 69: 1333-1338.

\section{*Correspondence to}

Yongchang Miao

The Second People's Hospital of Lianyungang'

Jiangsu Province

PR China 